\title{
Medea in Aracoeli? Nota (senza rete) per una possibile traccia mitologica in Elsa Morante
}

\author{
Marco CARMELLO ${ }^{1}$ \\ Universidad Complutense de Madrid \\ macarmel@filol.ucm.es
}

\begin{abstract}
RIASSUNTO
L'articolo intende verificare la presenza di una traccia mitica nell'ultimo romanzo di Elsa Morante, Aracoeli. La discussione metterà dunque in evidenza punti di contatto e divergenze fra il mito di Medea ed il romanzo morantiano, e cercherà di fornire una chiave di lettura nuova dell'opera.
\end{abstract}

Parole chiave: Morante, Aracoeli, mito, antropologia, Medea.

\section{Medea in Aracoeli? \\ A Note (without Protection) to Search a Possible Mythological \\ Mark in Elsa Morante}

\begin{abstract}
The article tries to define the presence of a mythological mark in Elsa Morante's last novel, Aracoeli. Therefore the discussion will highlight the connections and the deviations existing between the myth of Medea and the novel by Morante. We will try to define a new approach to Aracoeli.
\end{abstract}

Key words: Morante, Aracoeli, Myth, Anthropology, Medea.

Sommario: 1. Introduzione; 2. Considerazioni preliminari; 3. Strutturazione di un mito; 4. Mitologia e romanzo; 4.1 Prima considerazione breve: intorno al sopravvivere; 4.2 Seconda considerazione breve: intorno all'impossibilità di sopravvivere; 5. Conclusioni; Riferimenti bibiografici

1 Departamento de Filología Italiana, Facultad de Filología, Edif. D, Avenida Complutense s/n, Ciudad Universitaria, E-28040, Madrid, España. 


\section{INTRODUZIONE}

Che l'opera di Morante sia uno dei più complessi crogiuoli intertestuali del secondo Novecento è cosa nota, che, all'interno dell'opus morantiano, Aracoeli presenti una particolare difficoltà di interpretazione, è fatto altrettanto conosciuto; da queste due premesse segue una conclusione: l'ultimo romanzo non è certo il punto più agevole da cui iniziare un sondaggio dell'opera morantiana ${ }^{2}$.

D'altra parte però non si può non riconoscere che Aracoeli sia una sorta di raffinata cassa di risonanza, in cui convergono echi i più vari ed a volte inattesi che sia possibile, come se il romanzo volesse anche essere la definitiva prova di bravura di una scrittrice eminentemente intertestuale, la riprova ultima di come si possa fare della scrittura un sistema passante entro il quale la creatività autoriale si esercita anche come capacità di fusione fra echi, ipotesti, ipertesti, suggestioni, in un concerto equilibrato.

Perciò, pur se disagevole, Aracoeli resta la costa migliore, non solo rispetto a Morante, ma, più generalmente, rispetto al romanzo del secondo Novecento italiano, per iniziare - almeno idealmente - una possibile ricerca di quelle tracce mitologiche non dichiarate che possono tuttavia aver avuto un peso nella formazione di un universo poetico.

L'ipotesi di lavoro da cui parto è che sia possibile individuare una traccia del mito di Medea non solo nella creazione del personaggio eponimo del romanzo, Aracoeli appunto, ma anche nella strutturazione di alcuni tipi di rapporto che attorno al personaggio si costruiscono.

Cercherò, nelle pagine seguenti, di esporre le ragioni del mio approccio.

\section{CONSIDERAZIONI PRELIMINARI}

Romanzo di tinta eschilea, Aracoeli pone al lettore un problema immediato: da dove viene la voce narrante?

${ }^{2}$ La critica, non solo italiana, si è, nel corso degli ultimi vent'anni, esercitata sulle difficoltà, a volte sulle asprezze, dell'opera di Elsa Morante, dando così l'avvio ad una pregevole e, di anno in anno, sempre più fitta letteratura, per cui, oltre a quello pionieristico di Garboli, bastino i nomi di: D’Angli, Rosa, Zancan, Cazalè, Paduano, Mengaldo, Bazzocchi, Pischedda, Serkowska e Martínez Garrido. Chi scrive non appartiene, malgré soi, alla schiera dei "morantisti", di cui sopra, pur fra mille dimenticanze, si sono fatti alcuni pregevoli nomi. L'appunto che dunque l'autore si azzarda a proporre ha quindi un unico scopo: verificare se, entro quali limiti, con quali distinguo, sia possibile rintracciare un'eco mitologica precisa. 
Pischedda (2004, pp. 150-157) nota acutamente come il romanzo sia dominato da un cronotopo allucinatorio, che, nelle ultimissime pagine, porta Manuel, il protagonista/voce narrante di Aracoeli, quasi a fuggire dal piano cronologico della fabula, del resto difficilmente definibile nella sua meta-cornice di inquadramento, risultando così la sua voce del tutto sganciata dalla vicenda, ormai resa "monade solitaria", isolata dal flusso diegetico della narrazione.

Quel flusso stesso è ambiguo, sospeso nella cornice indefinita del viaggio di Manuel, costruito com'è intorno ad una concatenazione di rimandi, sospensioni, digressioni temporali e spaziali, in un continuo andirivieni fra la Milano dell'attualità, la Torino della repressione, la Roma ambigua dell'infanzia e della perdita dell'infanzia, ed infine l'Andalusia comunque fuori tempo di Aracoeli e del suo passato inviolabile.

Parrebbe quasi, se alla quidditas inerentemente illuministica del concetto non ostasse il netto rifiuto della Morante verso l'inganno "storico" della razionalità, che l'intera narrazione sia posta sotto il segno di un'insolubile rêverie ${ }^{3}$.

In realtà se quello di Manuel non fosse altro che una rêve, le pagine del romanzo avrebbero tutt'altro segno, non sarebbero invece poste sotto il richiamo di quella data, 1 novembre 1975, di cui si ignora se indichi una nascita o una morte, come non si sa se sia destino di nascita o di morte quello che Manuel incontra a El Almendral.

Manuel muore? O rinasce? O rinasce perché muore? Qualunque sia la soluzione - e non è fatto indifferente, poiché alle tre possibilità corrispondono tre letture differenti del romanzo -, è, per il nostro scopo, poco rilevante. In qualunque caso infatti, e torniamo ancora una volta a Pischedda, la narrazione viene da "fuori", dall'estraneità del narratore rispetto al mondo.

Aracoeli è veramente un romanzo "dalla tomba", da quell'aldilà, fatto di sepolture scoperchiate, come quelle del Verano sconvolto dai bombardamenti, da cui fugge ciò che impropriamente le lapidi mortuarie vorrebbero trattenere ed insegnare: l'essenza del vivere.

Senza tempo, perché fuori dal tempo, Manuel è quindi, ontologicamente e propriamente, in senso tecnico, una voce mitica.

Era importante fissare almeno questo punto di partenza per dimostrare quanto la dimensione mitica sia connaturata al romanzo; del resto, è anche questa "miticità" intrinseca di Aracoeli a renderne difficile la collocazione in un sottogenere preciso, non siamo infatti di fronte ad un romanzo familiare, o ad un Bildungsroman, a un romanzo storico, o ad un feuilleton, e neppure ad un romanzo di viaggio o ad un

\footnotetext{
${ }^{3}$ Il riferimento è ovviamente alla Poètique de la rêverie di Gaston Bachelard (1960), di cui troppo spesso si dimentica l'originale, sicuramente eterodosso, ma convinto, impegno razionalista, per parafrasare il titolo di una delle opere capitali del filosofo, epistemologo (ed ingegnere) francese.
} 
libro di memorie, anche se sarebbe facile dimostrare come il testo morantiano includa in sé caratteristiche di ognuno di questi sotto-generi.

Anzi, a tutti quei sotto-generi, ne vanno sicuramente aggiunti altri due: il romanzo psico-analitico, sulle tracce di quel breve scritto freudiano sul Romanzo familiare dei nevrotici ${ }^{4}$, di cui ha già ampiamente detto Giovanna Rosa (1995), e quello che potremmo definire "romanzo antropologico", in cui le opposizioni materno/paterno, genitori/figlio, femminile/maschile, generante (generatore)/generato, vitale/mortale, che definiscono intorno alle loro polarità l'intera vicenda narrata in Aracoeli, si fondono, quasi confusivamente, sull'opposizione straniero/autoctono, barbaro/civile, di questo luogo/di un altro luogo, parlante la mia lingua/parlante un'altra lingua - ossia, tout court, parlante/non parlante ${ }^{6}-$ che è di natura prettamente antropologica ${ }^{7}$.

L'opposizione antropologica su cui insiste l'architettura romanzesca è quindi quella primaria che determina l'interdetto: si tratta di quell'opposizione essenzialmente pre-politica, che fonda però il politico come spazio, in forza della quale si istituisce la linea di demarcazione fra fuori e dentro.

Per questo, forse, l'ultima opera di Elsa Morante è un romanzo anzitutto "spaziale", prima ancora che "temporale", basato sull'impossibilità del protagonista/narratore, Manuel, di trovare un suo reale ubi consistat, e sulla deprivazione locale, vale a dire sul bando, nel senso giuridico di atto di espulsione, che depriva del loro originario ubi consistant Aracoeli ed Eugenio Oddone Amedeo $^{8}$, padre e madre di Manuel.

Le considerazioni riguardo al luogo, o meglio alla struttura dell'interdetto che sembra presiedere la "località" in Aracoeli - si pensi solo a tutti i luoghi in cui

\footnotetext{
${ }^{4}$ La cui traduzione si legge alle pagine 469-474 dell'opera omnia di Freud (cfr. S. Freud, Opere: 1905-1908, vol. V, Torino, Bollati-Boringhieri, 1972).

5 Chiaramente opera qui l'eco della proposta semiologica di Greimas (1970), che istituisce una vera e propria teoria del racconto mitico. Sarebbe interessante, ma eccederebbe le pagine del presente lavoro, far reagire il romanzo di Morante con la proposta teorica del semiologo lituano/francese.

${ }^{6}$ Giova qui ricordare un fatto notissimo: barbaro è, nel senso etimologico, greco, originale, colui che balbetta, o meglio, colui che emette suoni incomprensibili, non linguistici, e sembra ripetere continuamente bar bar bar, l'onomatopea che i greci usavano per indicare quelli che, al loro orecchio, sembravano solo fonetismi disarticolati di un altro sistema non linguistico, e quindi propriamente animale, giusta l'equivalenza di linguistico ed umano svelata e definita da Aristotele.

${ }^{7}$ Come suggerisce Angela Di Fazio in un interessante ed utilissimo articolo (Di Fazio 2013). L'articolo di Di Fazio segue piste diverse da quelle qui percorse, anche se ha fornito non pochi spunti a queste pagine.

${ }^{8}$ Ignoro se la Morante ne fosse a conoscenza, tuttavia è interessante notare che il significato etimologico del nome Eugenio è "ben nato, di buona stirpe", Eugenio è cioè colui che nasce secondo le leggi da una buona famiglia.
} 
Manuel non può stare: Totetaco, i Quartieri alti, Torino, il Collegio, Milano - ci riportano a quell'essenza mitica del romanzo, di cui dicevamo poco sopra, permettendoci però adesso di iniziare ad individuare una mitologia precisa: quella di Medea, ossia quella della madre straniera, la cui prole, generata nella terra di nessuno del viaggio dalla terra paterna, da cui si è stati banditi, alla terra maritale, in cui non si sarà ammessi, non potrà trovare altro spazio che non sia quello, privo di territorio, dell'insistenza nel legame familiare.

\section{STRUTTURAZIONE DI UN MITO}

Il mito di Medea, almeno secondo i due testi che ne tramandano le versioni più note, vale a dire l'omonima tragedia di Euripide ed il III libro degli Arghonautikà di Apollonio Rodio ${ }^{9}$, è analizzabile nei suoi tratti salienti forse con maggior semplicità di quanto non si dia per altri miti.

La storia è nota: obbligato a recarsi nella lontana e barbara Colchide per recuperare il vello d'oro, che gli permetterà di riprendere il trono di Iolco, usurpato al padre Esone dal fratellastro Pelia, Giasone riesce nell'intento grazie all'amore di Medea, figlia del re della Colchide Eeta, che gli svela il segreto per impadronirsi del trofeo.

Inseguiti da Eeta i due riescono a sfuggire grazie ad un terribile stratagemma architettato ancora una volta da Medea, che uccide il fratello Apsirto facendone a pezzi il cadavere e gettandone in mare i singoli pezzi separatamente, in modo che Eeta, per recuperare e ricomporre la salma del figlio, debba rallentare il suo inseguimento, fino a recederne. Una volta giunti a Iolco, Medea ricorre ancora una volta ad un inganno, mediante il quale convince le figlie di Pelia a fare a pezzi il padre cuocendone le membra in un calderone; queste, illuse di dare così al loro padre l'eterna giovinezza, in realtà lo uccidono.

Esiliati, come istigatori dell'assassinio del padre, da Acasto, i due, Medea e Giasone, giungono finalmente a Corinto, dove Giasone si innamora di Glauce, figlia del re Creonte. Medea, sconvolta dalla volontà di vendetta verso Giasone, provocherà, con l'invio in dono di una veste avvelenata fatta consegnare a Glauce

9 Il mito giunge quindi alla nostra conoscenza già decantato da quello che viene comunemente, anche se impropriamente, chiamato "illuminismo greco", di cui Euripide fu forse il massimo esponente. Alla figura euripidea si sostituisce però presto quella forgiata da Apollonio, ulteriormente decantata nel complesso gioco ipo-/iper-testuale della poesia ellenistica, che offre un modello di riferimento i cui influssi più celebri, e più impattanti per la successiva tradizione, furono quello su Virgilio, che alla Medea di Apollonio si richiama nella costruzione della sua Didone, e su Ovidio, che riprende la figura sia nelle Heroides (Epistola XII) sia nelle Metamorfosi (Libro VII). 
dai suoi stessi figli, Mermero e Fere, la morte di questa e del padre Creonte; infine ucciderà di sua mano i figli suoi e di Giasone ${ }^{10}$.

Medea, che fugge da Corinto con l'aiuto di un carro inviatole dal nonno, il dio solare Elios, giungerà ad Atene, dove sposerà Egeo, ma anche da qui dovrà fuggire, aveva infatti tentato di causare la morte di Teseo favorendo così Medo, il figlio avuto con Egeo, per la successione al trono. Alla fine di tutta la sua vicenda Medea si riappacifica col padre, Eeta, e torna in Colchide.

Al contrario la vicenda di Giasone si conclude male: secondo alcune versioni l'eroe morirebbe all'istante per il dolore provocato dalla morte dei due figli, secondo altre invece, perso definitivamente il favore della sua protettrice Era e degli altri dei, Giasone muore, vecchio e solo, a bordo della nave Argo, con cui da giovane si era recato in Colchide.

Se adesso cerchiamo di ridurre ai suoi tratti essenziali il mito, possiamo abbastanza facilmente individuarne alcuni connessi sia alle singole figure sia al mito in generale.

Per quanto riguarda Medea risalta soprattutto un tratto caratteristico generale con cui potremmo lumeggiare il personaggio: quello del perturbamento erotico e dionisiaco. A-regolare, piuttosto che irregolare, l'estraneità di Medea assume un carattere agambenianamente ed ambiguamente sacro. La figlia di Eeta è travolgente nella sua attività puramente impulsiva, nella quale non si riconosce altro motore che non sia appunto l'impulso che la anima. Non bisogna dimenticare l'appartenenza di Medea alla stirpe del Sole ${ }^{11}$, segnata, come tutte le altre stirpi mitiche (Atridi e discendenti di Cadmo in testa), da una suo proprio destino di tracotanza, irregolarità, superbia, hubris, per usare il termine greco.

L'estraneità di Medea non è solo legata alla sua provenienza extra-ellenica, barbara, anzi si potrebbe quasi dire che la barbarie di Medea sia il contrassegno evidente di ciò che la mette costitutivamente fuori dallo spazio greco. Potremmo dire che tale caratteristica consista nella "forza naturale" di cui la maschera mitologica di Medea è portatrice, una forza che non può ricevere alcun segno di valenza da parte delle strutture della civiltà, non può essere né positiva né negativa.

Medea genera e distrugge, sostiene ed annulla, ama e si vendica, il tutto sempre entro il ciclo dionisiaco che la sorregge, che ne sorregge cioè quella "potenza" attuativa al di qua anche della più primitiva polarità, quella, generatrice di "civiltà", che oppone eros a thanatos.

${ }^{10}$ La narrazione delle parti precedenti del mito derivano da Apollonio Rodio ed Ovidio, quest'ultima riassume velocemente la Medea di Euripide. Per le successive la fonte è la Biblioteca del mitografo Apollodoro.

${ }^{11}$ La stessa di Circe, che le è zia per parte di padre. I singoli miti greci sono spesso riconducibili a "saghe familiari", a loro volta strettamente intrecciate fra di loro, ognuna delle quali sembra essere segnata da un preciso destino di Hubris. 
Medea è dunque il "perturbante/conturbante" (das Unheimliche) così come Giasone è il "perturbato/conturbato""

L'inevitabilità che sia Giasone il "colpito" dal conturbamento/perturbarmento è data dal tratto che più marca il personaggio maschile del mito di Medea.

La definizione del carattere fondamentale dell'eroe greco è più sfuggente: Giasone ha un rapporto profondo con la regolarità normante della civiltà, col nomos che regola e definisce l'esistenza umana, eppure questa relazione è ambigua. Dal momento della sua nascita, il percorso di Giasone è segnato da un destino di rivendicazione ed esclusione che ne fanno un arci-modello dello spazio politico greco e dei suoi mezzi di normalizzazione.

Costretto dall'usurpazione patita dal padre a rivendicare il suo legittimo diritto al trono - ad un trono che non avrà mai - l'eroe parte alla volta di un paese barbaro, che dovrebbe però fornirgli il mezzo di quella legittimità politica cui aspira. Il nesso esilio/rivendicazione diventa allora chiaro: solo riuscendo ad entrare in contatto con quel primordiale assoluto che permette la vita è possibile correggere l'ingiustizia cui lo spazio interno del politico ha sottoposto Giasone.

Se il nesso è chiaro non meno lo è il modo: Giasone dovrebbe controllare la forza perturbante/conturbante di Medea, dovrebbe cioè essere possibile la costruzione di una soglia condivisa fra regolarità dello spazio cittadino di Giasone $\mathrm{e}$ a-regolarità dell'estraneità di Medea. Il mito è l'immagine del fallimento di questo processo.

Adottando un approccio à la Untersteiner (1991 [1946]), possiamo leggere l'intero mito di Medea come lo scontro inconciliabile fra politico e pre-politico, interno segnato dalla necessità di regolare la vitalità ed esterno marcato dall'insopprimibile bisogno di vitalità pura, impassibile di regolazione ${ }^{13}$.

Il punto di rottura dialettico è rappresentato dall'uccisione dei figli, impossibili, di Medea e Giasone.

Mermero e Fere sono personaggi muti, puri mezzi subordinati della lotta del vitale/Medea contro il regolarizzante/Giasone, sono strumenti della madre che cadono nella zona d'ombra della disattenzione paterna. Come spesso accade sono proprio i personaggi non agenti, quelli che l'economia mitica trasforma in puri pazienti, a rivelare il senso profondo di una vicenda mitologica.

12 Segnalo, lasciando che il lettore ne tragga le conseguenze, lo scivolamento del concetto di conturbante/perturbante verso una sfera di antropologia filosofica che riavvicina il lessico psicoanalitico alla sua originaria valenza mitografica.

${ }^{13}$ In questo senso possiamo dire che al mito di Medea si oppone quello di Antigone. Mentre il primo contratta a monte lo spazio politico, e consiste nello scontro fra ciò che si può includere e ciò che si deve escludere, il secondo è tutto interno allo spazio politico e la sua posta consiste in chi e come debba definire la norma. Non a caso il mito di Medea riguarda la generazione, o meglio la sua impossibilità, mentre quello di Antigone si riferisce alla morte ed alla sua regolazione. 
I figli non hanno una marca loro propria, ma, con la loro vicenda, segnano il senso dell'intero mito. La loro generazione è impossibile, impossibile perché è l'effetto irregolato di un eccesso di forza vitale, quella stessa forza vitale che può portarli alla luce ma non può preservarli laddove la normazione politica, legale, della razionalità regolare non la obblighi a lasciare spazio, cioè a ritirarsi perché possa esistere quello spazio necessario a che si crei l'ubi consistat del generato.

La regola della città però, come ci insegnano le azioni di Giasone, incapace o ingannatore, esclude e travia, non mantiene $\mathrm{i}$ patti con la terrestrità della forza vitale, non diventa insomma nomos, legge; pone i figli barbari di Medea in balia di Medea, che non può che travolgerli nella sua sfrenatezza erotica verso Giasone.

Il mito di Medea è quindi al fondo la tragedia del generato che ha scoperto la prova provata dell'impossibilità della sua esistenza, dell'annichilazione di senso della sua generazione, ed è proprio questo, come vedremo, il punto di contatto con Aracoeli, a sua volta romanzo dell'insensatezza generazionale.

\section{MITOLOGIA E ROMANZO}

Un mito contiene molto di più di quanto si riesca a dire, ma soprattutto un mito è una meta-narrazione, un serbatoio di temi, o di archetipi, se si preferisce, da cui poi il narratore trae ciò che serve alla sua narrazione.

Medea travalica Aracoeli, come il mito travalica la narrazione - lo si è appena detto - ma ciò che importa è stabilire cosa vi sia di Medea in Aracoeli.

Le assonanze esteriori sono molteplici: Aracoeli è una straniera impresentabile, giunge da una terra estrema, ignota, marcata, rispetto alla centralità della Roma dei quartieri alti e della Torino dei nonni, da una componente irrimediabilmente barbarica ${ }^{14}$.

La barbaricità dell'originaria provenienza di Aracoeli ritorna del resto nei deliri tossicomani del Manuel maturo, che, immaginandosi sotto processo, fa dire alla sua difesa, commentando l'ingenuità delle credenze religiose che madre e figlio condividevano ai tempi della loro vita fusionale nel popolare quartiere romano di Monte Santo, la Totetaco dell'infanzia del protagonista:

D. Conviene rammentare che la madre del Soggetto era andalusa. Andalusia - Andaluz già detta Spagna musulmana. Semprevergine è detta Maria nelle Beatitudini, ma così

${ }^{14}$ L'estraneità mitologica e barbarica, positivamente marcata per il Manuel bambino e ragazzo, mentre è negativamente giudicata dalla famiglia paterna, è evidente fino alla fine del romanzo, quando Manuel maturo finalmente giunge a quella terra per scoprire che è, esattamente come tutte le altre, deserto. Significativamente l'Andalusia originaria sembra non esistere solo per Eugenio, l'unico personaggio rispetto cui il territorio di provenienza di Aracoeli non riceve alcun segno. 
anche vengono dette le Urí nel Paradiso Islamico. È possibile che in quella madre andalusa sopravvivessero teofanie ancestrali d'Arabia e d'Africa. Nel suo cattolicesimo elementare, tornavano rituali barbari e favole pagane (Morante 1990, 1185).

L’appartenenza di Aracoeli al mondo barbaro è qui esplicitata attraverso il sistema credenziale di cui ella è portatrice: come la maga Medea, anche la madre andalusa è fonte di un sapere altro, più antico, ma proprio per questo squalificato, inaccettabile rispetto alla civiltà paterna. È un sapere non solo inutile, ma, esattamente come quello di Medea, pericoloso, potenzialmente distruttivo.

Questa perniciosità è indirettamente esplicitata nel delirio di Manuel, nel quale Morante sembra riassumere il senso dell'intera esperienza infantile del protagonista, segregando però gli anni di Totetaco in un limbo dislocato al di qua dell'opposizione vero/falso ${ }^{15}$. Significativamente è la "difesa" di Manuel a evidenziare la traccia "barbarica" che il protagonista condivide con la madre, ricordandoci così uno dei motivi essenziali di quell'estraneità che segna Manuel, rendendolo disadattato rispetto al mondo. La traccia barbarica di Aracoeli, venuta per mare, nascosta su di una nave, da un terra lontana, determina il figlio, avvicinandolo alle radici del mondo mitico, infantile, ma impedendo, proprio per via dell'eccessiva vicinanza, una ri-significazione di quel mondo all'interno delle strutture di conoscenza della "civiltà"16.

La barbaricità di Aracoeli si esprime però anche più propriamente: la protagonista non sa parlare né scrivere, al punto che l'intero periodo di Totetaco è segnato da un tirocinio che ha proprio nel congiunto apprendimento linguistico di madre e figlio il suo punto focale. È interessante notare come Morante incroci il piano linguistico: Aracoeli non apprenderà mai bene l'italiano, continuando a fluttuare in un'interlingua sconvolta, fatta di spagnolo a malapena italianizzato ${ }^{17}$, mentre a Manuel, destinato a diventare abile possessore del gioco linguistico, l'unica lingua preclusa sarà proprio lo spagnolo, quella che, tecnicamente, avrebbe dovuto essere la sua lingua madre. I due risultano così inchiodati nell'impossibile,

${ }^{15}$ La funzione di questo "processo" finzionale, che rappresenta una delle tante "soglie" del romanzo, andrebbe meglio valutata in relazione a quei "cortocircuiti" spazio-temporali che sorreggono l'intera struttura di Aracoeli.

${ }^{16}$ Non a caso il delirio/processo di Manuel ha come posta in gioco il rifiuto di una interpretazione psicoanalitica dei suoi ricordi infantili, che ha lo scopo di salvaguardare l'estraneità dell'intero periodo di Totetaco rispetto alle strutture significanti dei Quartieri Alti, della zia Monda, dei nonni di Torino.

${ }^{17}$ L'esempio più drammatico e rilevante è il biglietto di addio al marito: "ANDATA VIA PARASIEMPRE FINNITO IO/ NO SONO DIGNA DESER TUA ESPOSA IO DISSONORATA/ NO CERCARME NUNCA NINGUNO NESUNO NO CERCARME NO/ ESPERARME/ NO NO ASI POTESI DARTI TANTI BACCI/ FINNITO ADIO/ ARAC»(Morante 1990, 1384). 
ed inappartenente, lingua panica ${ }^{18}$ di Totetaco che impedisce quel dominio dei codici linguistici di cui abbiamo appena detto.

La barbarie linguistica di Aracoeli, sia essa la dolce inciviltà dei «fiorisol», dei «fioripaloma», dei «fioriluna», oppure quella aspra dell'addio al marito, è anche segnale di come ella appartenga a quel mondo della "forza naturale", irregolabile, di cui parlavamo a proposito di Medea.

Come sappiamo l'irregolabilità di Aracoeli esplode nella parte finale del romanzo, travolgendola in una sorta di ninfomania sacra che ha, sulle strutture della civiltà di appartenenza, accuratamente riflesse nell'organizzazione familiare da cui discende ed a cui appartiene Eugenio, un effetto dirompente tale da essere, nella sua portata, paragonabile all'infanticidio di Medea.

In realtà, come si dirà fra poco, Aracoeli compie, nella persona di Manuel, un vero e proprio infanticidio traslato, che non trova alcun freno da parte di Eugenio, la cui inadeguatezza paterna ${ }^{19}$, oltre ad avere un senso di fratellanza profonda con Manuel (come ben rileva Bazzocchi 2009), reso chiaramente esplicito dalla stessa Morante $^{20}$, può però avere anche un'altra, complementare, chiave di lettura.

La fascinazione di Eugenio per Aracoeli è completa:

Essendo lui represso da sempre nella natura, i suoi moti festanti somigliavano a quelli, maldestri, di certi cuccioli di cane. Ma quando mia madre e lui si allacciavano stretti nel benvenuto, il loro amore si scioglieva dall'impaccio in un frullo gioioso, che faceva tremolare la luce. (Morante 1990, 1086)

Credo che questo semplice passaggio renda evidenti i rapporti fra i due mondi: Eugenio scopre in Aracoeli e nel suo amore la liberazione di quella "repressione nella natura" di cui la coppia-modello dei nonni torinesi, i genitori di Eugenio, rappresenta il prototipo.

Come nel caso di Giasone la fascinazione è totale, come nel caso di Giasone è tale da portare il personaggio a perdere l'appartenenza al suo modo definitorio, e conseguentemente a non potersi opporre alla catastrofe che distruggerà quel mondo, come nel caso di Giasone, anche per Eugenio la catastrofe provocata da Aracoeli/Medea, travolge il personaggio col suo valore apocalittico di disvelamento

18 «Bisogna dire che esisteva una parentela stretta fra tutte le cose: tutte apparentate dalla luce» (Morante 1990, 1183).

19 «Verso di me, fino da principio, i suoi modi accusavano, insieme a una buffa imperizia del mestiere di padre, anche una discrezione esitante e confusa, quasi temesse di impormi la sua podestà, sotto qualsiasi aspetto» (Morante 1990, 1206).

20 «Fra me e lui, corse subito - in luogo dell'affetto carnale - una silenziosa concordanza: forse in virtù della nostra comune passione per Aracoeli. È certo che il nostro amore grande, esclusivo per la stessa donna era fra noi due motivo di riconoscenza, piuttosto che di contesa» (Morante 1990, 1206-1207). 
della menzogna insita nella legge paterna ${ }^{21}$, eppure è proprio nel personaggio di Eugenio che si opera il maggior scarto rispetto alla vicenda mitica.

Si tratta di una scarto fondamentale sia dal punto di vista dell'economia narrativa sia da quello della costruzione dell'universo concettuale del romanzo morantiano: Eugenio è fedele ad oltranza ad Aracoeli, anzi, Morante costruisce l'intero personaggio intorno a quest'indefettibile fedeltà; così, se anche Eugenio accoglie in se quasi tutti i tratti del Giasone mitico, egli è però privo del tratto demarcatore essenziale di Giasone, la rottura della fedeltà giustificata ex lege $e^{22}$.

Questa fedeltà, che pone Eugenio fuori legge, almeno secondo le regole del suo gruppo, ha un effetto essenziale anche per quanto riguarda Aracoeli. A differenza del personaggi mitico, Aracoeli è definitivamente esiliata dalla sua patria, destinata a morire, distruttrice autodistrutta, nella capitale del paese di quel marito che è però "nemico" dei suoi: non si dimentichi infatti che il fratello Manuel - non casuale omonimo del figlio - cade per la Repubblica Spagnola, lottando contro i fascisti sostenuti, fra gli altri, anche dalle forze armate del Regno d'Italia.

Il fratricidio di Aracoeli, sebbene metaforico, ha dunque conseguenze più profonde rispetto a quello reale di Medea, a provarlo vi è la figura distante della madre di Aracoeli come la vede Manuel al capezzale della sua propria madre. La «nonna di Spagna»: «...si ritraeva tutta verso il muro: non per umiltà sociale sembrava - ma per una specie di apartheid volontario, quasi scostante» (Morante 1990, 1416).

Per Aracoeli non è più possibile tornare alla sua patria, come lo è ancora per la Medea mitologica, resta in una sorta di terra dell'esilio, catturata e fermata dall'amore coniugale di Eugenio, vero e proprio specchio di Giasone, poiché agisce in maniera simmetrica e contraria rispetto a quella dell'eroe greco.

La portata di questo scarto è dunque ampia, e ha, a giudizio di chi scrive, una ragione ben precisa: se il mito di Medea era il mito che inscenava il contrasto fra politico e pre-politico sotteso all'atto del generare, il romanzo di Morante è invece interamente incentrato sulla figura del generato, del figlio. Questo cambio di prospettiva è quindi funzionale alla costituzione della figura di Manuel, protagonista e narratore al contempo, intorno a cui tutte le tensioni che si

${ }^{21}$ Che, nel caso di Eugenio, coincide anche col tradimento storico effettivo di Vittorio Emanuele III, ossia dell'ipostasi del "padre" nell'economia regolativa del personaggio: «[...] il suo massimo riguardo - direi meglio il suo culto - era votato al re [...]» (Morante 1990, 1084).

${ }^{22}$ Essendo Medea una barbara, il suo legame con un greco non poteva, secondo le leggi poliadi generalmente vigenti in Grecia, essere considerato valido a tutti gli effetti, né i figli della coppia erano pienamente legittimati; non a caso è proprio la situazione legalmente, e politicamente, incerta dei figli l'argomento usato dal Giasone euripideo nella rhesis con cui tenta di convincere Medea della giustezza del suo agire (vv. 522 - 575). 
definiscono in Aracoeli finiscono per addensarsi. Prima quindi di giungere alle conclusioni, bisogna dedicare due considerazioni a Manuel.

\subsection{Prima considerazione breve: intorno al sopravvivere}

Il personaggio/narratore che incontriamo all'inizio del romanzo è segnato da una sorte chiara: Manuel, ricostruendo il destino che gli è stato dato assegnato al momento della nascita dice di se stesso:

[... ] fra le sorti indelebili della mia trama futura, ormai cucite dentro la mia carne, la prima diceva: MAI PIÚ TU SARAI/ UN OGGETTO D'AMORE/ MAI PER NESSUNO MAI/ MAI TU SARAI UN OGGETTO/ D'AMORE (Morante 1990, 1094).

Manuel, il Manuel maturo che sta all'inizio della narrazione riconsiderando l'intera sua storia di vita, ci appare come un escluso che sopravvive fuori dal fondamentale legame unitivo umano, quello dell'amore.

Dovremo aspettare la fine della narrazione per ritrovare il momento che definisce, nel senso di un vero e proprio infanticidio traslato, quest'esclusione. La scena è una delle più note del romanzo: immediatamente dopo il tentato suicidio dell'attendente del padre, Daniele, sconvolto dal tentato adescamento di Aracoeli, assistiamo ad una porzione di giornata condivisa fra madre e figlio, che, dopo un tesissimo pranzo cui partecipa la zia Monda, usciranno per andare in chiesa, dove Aracoeli renderà definitivo l'allontanamento del figlio da sé. La pericope narrativa si conclude con l'ingresso di Aracoeli e Manuel in chiesa, dove l'impurità/estraneità della protagonista deflagra.

L'intera seconda parte di questa sezione è dominata dal suono in sottofondo del campanello del gelataio ambulante verso cui Aracoeli spinge Manuel, suono che segna, almeno così mi sembra, il compiersi dell'infanticidio dello stesso Manuel ${ }^{23}$.

Interessano in questa sede due passaggi: il primo, subito dopo il pranzo, segna la repulsione di Aracoeli verso il figlio:

[...] lei pure ebbe un accenno di riso ... ma subito lo spense, riguardandomi, invece, ancora una volta con quei due occhi spalancati che parevano, al guardarmi, vedere in luogo di me un'ombra enorme; e poi subito si rinserravano con forza, in una cecità distruttiva. "Mama", io le dissi toccandole il vestito; e in risposta lei gridò: "Non toccarmi! Hai le mani sporche!" con una voce innaturale e stralunata.

Io mi guardai le mani. "Hai le mani sporche! Hai le mani sporche!" lei si accanì a ripetere, con una voce strana, piccola ma urlata, in un tumulto vorticoso dei nervi.

${ }^{23}$ Siamo del resto in un contesto di parodia religiosa, per cui al campanello del carretto sembra corrispondere quello che durante la messa cattolica segna l'avvenuta consacrazione dell'eucarestia, rinnovando il momento in cui avviene il sacrificio del Cristo, a cui è, in qualche modo, simmetrico quello di Manuel. 
"Vàttene!" continuò, "non devi più guardarmi! Non devi più toccarmi! Sei sporco! Sei brutto! Vàttene!’[...] (Morante 1990, 1374).

Il secondo passaggio narrativo invece si trova all'inizio della seconda pericope: la sequenza è iniziata proprio dall'apparizione del carretto del gelataio. Riporto di seguito, ampiamente compendiata, la scena che qui ci interessa:

[...] Allora lei mi disse piano, come un bacio nell'orecchio: "Manuelito! Manuelito!". E portando le mie proprie mani sulle proprie guance, e premendole con le sue palme, mi domandò, con una voce piccola e forastica, tintinnante come un vetro frantumato:

"Sei sempre il mio niño? Sei sempre il niño mio?"

La mia risposta era là pronta, ma lei brusca la scansò, liberandosi le guance dalle mie mani, nell'atto di chi si strappa una benda. E proferì con un'altra voce, indurita, piatta e sconciata come da un'operazione feroce di restauro:

"E invece, io non sono più la tua mamita" [...] (Morante 1990, 1377).

Manuel, sospinto quasi con cattiveria da Aracoeli, corre verso il carretto nell'estremo tentativo di compiacere la madre. Giunto a metà strada, il bambino si accorge di non avere i soldi per il gelato, quindi torna indietro per chiedere alla madre il denaro, ma:

[...] vidi che lei nel frattempo, s'era abbandonata con la testa fra le braccia, sui ginocchi ripiegati, come vinta da spossatezza o da sonno. Quando le fui vicino, tuttavia, levò il capo inaspettatamente e disse: “Andiamo! Andiamo è ora!" senza più ricordarsi del gelato.

Pure nel suo semplice dire: "andiamo andiamo" essa aveva ostentato un tono bizzarro, fra di capriccio e di bestemmia, o addirittura di delitto [...] (Morante 1990, 1378-1379).

I due si avviano quindi alla chiesa dove, davanti alla verginità di Maria, avviene la finale esposizione dell'impurità, sessualmente marcata, di Aracoeli ${ }^{24}$.

Non credo che i due passaggi riportati necessitino di molti commenti: qui vi è tutto il potere di Aracoeli, quella "forza naturale" da cui è travolta, e attraverso cui lei travolge chi le è intorno. Ma soprattutto qui c'è il senso più profondo dell'infanticidio: chi ha generato ora nega la generazione, escludendo il generato da quel legame d'amore senza il quale si precipita nudi "in un universo indifferente". Paradossalmente a questo punto l'unica speranza di Manuel sarebbe che Aracoeli attuasse fattualmente quell'infanticidio che è invece reso ancor più terribile dal traslato implicito nella sua metaforicità, per questo Manuel pregherà il fantasma della madre nell'unico modo che abbia un senso: «Ma tu, mamita, aiutami. Come

${ }^{24}$ Quest'opposizione Maria/Aracoeli, che si consuma nella città in cui vi è la Basilica di S. Maria in Aracoeli, mi pare una chiave non indifferente nella lettura del personaggio femminile della storia. 
fanno le gatte coi loro piccoli nati male, tu rimàngiami. Accogli la mia deformità nella tua voragine pietosa» (Morante 1990, 1174).

\subsection{Seconda considerazione breve: intorno all'impossibilità di sopravvivere}

A Manuel, orami irrimediabilmente marcato come brutto, sporco, portatore quindi di un'impurità, quella di colui che ha ricevuto da colei che lo ha generato lo stigma di chi non avrebbe dovuto essere generato, non resta che sopravvivere. Ma la sopravvivenza è data solo se la vita è possibile, mentre i nonni di Torino negano a Manuel esattamente la possibilità del suo vivere.

Bisogna intendersi bene sul senso di "dovere" e su quello di "potere"; se, come già si è detto, è Aracoeli a negare la ragione ultima della vita di Manuel, ponendolo definitivamente al di fuori del legame affettivo, non è però Aracoeli a chiudere la possibilità di sopravvivenza.

Il gesto di Aracoeli ri-toglie a Manuel la vita che non gli era dovuta, ma Manuel potrebbe continuare a sopravvivere se fosse possibile accettarne la presenza all'interno del quadro regolativo della legge paterna, se cioè fosse possibile a Manuel passare dalla dimensione mitica della sua generazione a quella storica, inserendosi così nella società paterna.

Proprio questa possibilità gli è tolta dai Nonni torinesi, che, in forza di quello scarto detto sopra, per cui Eugenio è lo specchio invertito di Giasone, è una specie di Giasone che si conserva ostinatamente fedele alla sua "Medea", divengono gli operatori di quel rifiuto secondo legge della prole irregolare che, nel racconto mitico, viene compiuto dall'eroe di Fere.

Bisogna infatti dire che, nel mito greco, il venir meno della fedeltà alla sposa è anche il venir meno della fedeltà ai figli, ossia l'annullamento del legame paterno ${ }^{25}$. Se ripensiamo al discorso con cui Giasone cerca di convincere Medea della "giustezza" delle sue nozze con Glauce, figlia del re di Corinto ${ }^{26}$, individuiamo immediatamente nella "salvezza dei figli" uno degli assi portanti.

Ancora una volta, secondo la logica del ribaltamento che la conservazione della fedeltà di Eugenio verso Aracoeli comporta, assistiamo ad uno scarto rispetto alla vicenda di Giasone e Medea. In quest'ultima era il venire meno della parola dell'eroe a negare implicitamente l'appartenenza poliade dei figli, causandone indirettamente l'assassinio, qui invece l'esclusione deve essere ancora pronunciata, e pronunciata nel senso di una impossibilità per Manuel di appartenere alla "civiltà" del padre.

${ }^{25}$ Legame che non sussisteva, essendo secondo legge i figli di Medea "bastardi" di Giasone ed impossibilitati perciò ad entrare, a pieno titolo, nella comunità poliade.

${ }^{26}$ Il riferimento evidente è alla Medea di Euripide (si consideri tutto il secondo stasmo della tragedia, vv. 446-626). 
Non è dunque il padre, ma è piuttosto il "paterno" a definire quella condanna: spetta quindi alla coppia normativa modello, rappresentata dai Nonni, la definizione e la pronuncia dell'esclusione di Manuel, esclusione che rende al protagonista realmente impossibile incontrare un luogo entro cui possa darsi la sopravvivenza.

Il passaggio che apre il racconto delle vicende di Manuel a Torino è, a riguardo, tanto chiaro, da meritare che se ne riportino le prime righe:

Il primo fenomeno che segnò, fino dall'inizio, il mio soggiorno presso i nonni, fu la balbuzie, dalla quale io guarii soltanto alla loro morte. A me i due vecchi si presentavano come una sorta di sdoppiamento della statua parlante del Commendatore: solo che io non avevo né le virtù di Don Giovanni, né quelle, complementari, di Leporello (Morante 1990, 1399).

Significativamente la prima reazione di Manuel è proprio la balbuzie, ossia il tratto che etimologicamente contraddistingue il barbaro, vale a dire il "balbuziente", colui che emette suoni inarticolati non linguistici. È una balbuzie che continua fin tanto che Manuel è esposto alla vista dell'occhio della legge ${ }^{27}$, che durerà fino alla morte dei due guardiani. La morte non è però, in questo caso, salvifica, è questo il senso del richiamo mozartiano: fusi nell'immagine fatale, ed inevitabilmente mortifera, del Commendatore, i Nonni rappresentano la condanna senza appello di Manuel, come appunto il Commendatore suggella la caduta infernale di Don Giovanni.

Di fronte a questa condanna Manuel è completamente nudo; non avere né le virtù di Don Giovanni né quelle di Leporello significa infatti che il nostro protagonista non può né decidere di assumere su di sé la condanna, mettendone in atto il dispositivo ma rimanendo fedele alla sua propria volontà - come Don Giovanni $^{28}$-, né rientrare nel limitato ambito di una sopravvivenza scaltra ed accorta ma ostinatamente ossequiente alla "legge" - come sarà poi quella di Leporello $^{29}-$.

Sacrificato ed "ucciso" da Aracoeli/Medea, il sopravvivente Manuel non può che essere bandito "secondo legge", ed ancora una volta il bando viene pronunciato per bocca femminile durante una cena ${ }^{30}$ :

\footnotetext{
${ }^{27}$ Non a caso Morante decide che la professione del Nonno di Torino sia proprio quella di magistrato e giurista.

${ }_{28}$ Perché Manuel potesse scegliere questa strada, avrebbe dovuto non essere sacrificato dalla madre.

${ }^{29}$ È proprio questa l'opzione che il "paterno" nega a Mozart.

${ }^{30}$ Come nel caso di Don Giovanni, in cui la condanna è connessa con un invito a cena. Del resto i due, Don Giovanni e Manuel, non sono altro se non "poveri Cristi".
} 
Fino da Totetaco, anch'io piccoletto con Aracoeli, ero stato debitamente istruito dalla zia Monda sulle norme dell'etichetta a tavola. Ma al cospetto dei Nonni capitava che non soltanto il mio cervello, ma gli stessi miei muscoli soffrissero di amnesia. Scombinato, m'imbrogliavo peggio di un bambinello. Mangiando il pollo con le mani, o attaccando la frutta a morsi, o succhiando gli spaghetti che mi pendevano lunghi giù dalle labbra. Poteva succedermi di sporcarmi di sugo fino sul naso; e poi di pulirmi col lembo della tovaglia. E nell'aspirare le cucchiaiate di minestra (evitavo di inghiottirle, per via del brodo bollente) fatalmente mi sortiva un sibilo. "Ma il cucchiaio, è forse un fischietto?!" "Ma qual è, la tua destra? E la tua sinistra?" "Ma gli occhiali, a che cosa ti servono?". In certi casi, la Nonna, rinunciava a qualsiasi commento, scambiandosi col Nonno uno sguardo costernato di fronte alla mia corruzione irrimediabile; ma in tale astinenza forzata, visibilmente si straniva, finché una sera (che i miei sbadigli - temo - non si contavano) d'un tratto m'investì. "Ma chi t'ha avvezzato a questi comportamenti a tavola? Sei forse cresciuto fra i barbari? O fra i cani di strada? Certo" soggiunse, "non è certo da nostro figlio, che imparasti...". (Morante 1990, 1408)

Anche in questo caso il testo è tanto trasparente da non richiedere lunghi commenti, mi interessa però far notare alcuni aspetti: anzitutto, il bando di Manuel, la sua ascrizione definitiva al mondo barbarico e, quindi, la sua conseguente espulsione da quello paterno (" 'Sei forse cresciuto fra i barbari? O fra i cani di strada? Certo' soggiunse, 'non è certo da nostro figlio, che imparasti...' »), avvengono a tavola. La cosa è importante per tre ragioni: per il valore metaforico che la cena ha nella nostra cultura; perché a Manuel viene negato il diritto di accostarsi al cibo, quindi gli viene metaforicamente negata la sopravvivenza; perché il valore ideologico delle "buone maniere" a tavola è uno degli aspetti più importanti per la definizione dell'appartenenza antropologica ad un gruppo ${ }^{31}$.

L'esclusione di Manuel è a questo punto completa: messo fuori dal vincolo affettivo e da quello normativo, non gli rimane altro se non scivolare lungo il crinale di un'esperienza ormai puramente testimoniale.

31 Si ricordi che nel 1971 usciva la traduzione italiana del terzo volume delle Mythologiques di Claude Levi-Strauss, ossia L'origine delle buone maniere a tavola (originale francese del 1968: L'origine des manières de table), libro noto nei circoli culturali che Morante frequentava, in cui Levi Strauss descrive le buone maniere a tavola a partire dal loro valore di denotatore culturale. Che le buone maniere siano uno dei mezzi centrali di definizione dell'identità culturale occidentale, lo dimostra anche un'opera fondamentale di Norbert Elias, ossia il primo volume di quell' Über den Prozeß der Zivilisation, che risale nell'originale versione tedesca al 1939, interamente dedicato alla nascita delle buone maniere (la traduzione inglese è del 1968, quella italiana, cui si fa qui riferimento, è dell'82). 


\section{CONCLUSIONI}

Bazzocchi (2004: 153) nota come l'ultimo periodo produttivo di Morante si avvicini, nella profonda disillusione verso l' "innocenza", all'ultimo periodo produttivo di Pasolini, nonostante un certo apparente allontanamento (per cui cfr. Siti, 1994) intervenuto fra i due. Come Pasolini, anche Morante sembra giungere ad esiti di lucida, ma chiusa, disperazione.

Il passaggio dalla storia al mito, il mito di cui ho cercato di ricostruire le tracce in Aracoeli, segna anche un passaggio dal romanzo delle generazioni, nella cui cornice storica trova ancora spazio la dinamica manzonianamente salvifica della "provvida sventura", al romanzo del generato, che si colloca invece oltre la storia, in un territorio che non può ricevere luogo, secondo quanto appunto diceva Pischedda (2004) riguardo all'impossibilità di localizzare il dove da cui proviene la voce narrante di Manuel nelle ultime pagine del romanzo.

Il passaggio dalla storia al mito è però anche il passaggio dalla dialettica all'aporia, secondo quell'interpretazione "fisiologica" à la Untersteiner, per cui il mito è l'inscenamento di un conflitto insolubile.

Lo "scandalo" della storia poteva ancora, nell'ottica di Morante, essere quasi cristologicamente salvato dalla "passione" delle vittime, che raggiunge in Useppe la dimensione piena dell'olocausto; non può invece trovare soluzione la condizione umana, vale a dire quel portato pre-storico che sempre determina la storia. Questa condizione, che agambenianamente si potrebbe definire "di nudità", trova nel male dell'essere generati il suo stigma.

La generazione stessa è male, perché ci espone ad una vita che ci obbligherà al male, rivelandoci così l'essenzialità della malvagità, della colpevolezza, di quello che i greci chiamavano miàsma - contaminazione - per la definizione della nostra vita.

Manuel è sacrificato, ma a differenza di Useppe, non è vittima, ma simbolo: è impuro, contaminato, radicalmente corrotto perché all'origine impura e radicalmente corrotta era la sua stessa vita. È qui all'opera una logica del mito, che domina tutto il testo di Aracoeli, in forza di cui ogni gioco di rapporti è a somma zero, poiché la questione della colpa non si pone, nel mito, singolarmente, ossia moralmente, ma complessivamente, quindi ontologicamente.

L'unica via di uscita è perciò l'annullamento (cfr. Bazzocchi 2004): la vera salvezza, per Manuel, è diventare voce narrante, diventare cioè egli stesso attività mitica, narratore, che si pone, rispetto all'evento mitologico e narrato, al di là; non per forza in un al di là di morte, ma sicuramente in un al di là in cui vi sono le chiavi, linguistiche e narrative, dell'uscita dall'aporia insanabile dell'essere generati.

Solo dopo aver raggiunto queste chiavi, solo dopo aver cioè espugnato l'ultimo baluardo mitico che Manuel ostinatamente cerca di difendere, fino cioè al suo arrivo nella sassaia desertica di El Almendral, dove finalmente appare chiaro che «non c'è niente da capire» (Morante 1990, 1428), sarà possibile la soluzione dell'aporia. 
Solo a questo punto la voce ormai mitopoietica di Manuel può ricongiungersi con quella, silenziata per tutto il corso della narrazione, puramente umana, anch'essa "nuda", dell'unico personaggio che, conservando la "fedeltà d'amore", esce dallo schema del mito: Eugenio Amedeo Ottone. È forse in questa fusione di padre e figlio, generato e generatore, umanità e voce mitopoietica del narrante, che Morante indica l'unica via d'uscita possibile dal "male di vivere".

\section{RIFERIMENTI BIBLIOGRAFICI}

\section{Fonti primarie}

APOLLONIUS RHODIUS (1970): Argonautica, recognovit brevique adnotatione critica instruxit Ermannus Frankel, Oxonii, Typographeum Clerendonianum. [Traduzione italiana di Guido Paduano: Le Argonautiche, introduzione e commento di Guido Paduano e Massimo Fusillo, Milano, Rizzoli-BUR, 1986]

EURIPIDES (1984): Medea, in: Euripidi fabulae, tomus I, quo insunt: Alcestis, Medea, Heraclidae, Hyppolitus, Andromacha, Hecuba, recognovit brevique adnotatione critica instruxit Iacobus Diggle, Oxonii, Typographeum Clarendonianum. [Traduzione italiana di Umberto Albini: Medea - Ippolito, introduzione e traduzione di Umberto Albini, note di Maurizia Matteuzzi, Garzanti, Milano, 1990]

FreUD, Sigmund (1931): Der Familienroman der Neurotiker, in: Freud, Sigmund, Schhriften zur Neurosenlehere und psychoanalytischen Technik, Wien, Internationaler Psychoanalytischer Verlag. [Originariamente apparso, sine titulo, in: RANK, Otto (1909) Der Mythus von der Geburt des Helden, Leipzig und Wien, Deuticke: 64 - 68. Traduzione italiana di Marilisa Tonin Dogana: Il romanzo familiare dei nevrotici, in: Freud, Sigmund: Opere. Volume quinto, 1905 - 1908, edizione diretta da Cesare Musatti, Torino, Bollati - Boringhieri, 1972: 469 - 474]

MoRANTE, Elsa (1990 [1982]): Aracoeli, in: Opere, vol. II, a cura di Carlo Cecchi e Cesare Garboli, i Meridiani, Milano, Mondadori: 1037-1454. [Edizione originale: Torino, Einaudi, 1982]

PuBlius OVIDIUS NASO (1960-1962): Les Metamorphoses, texte établit et traduit par George Lafaye, Collections des Universités de France, Paris, Les Belles Lettres. [Traduzione italiana di Enrico Oddone: Le Metamorfosi, Milano, Bompiani, 1988]

Publius Ovidius Naso (1898): Publi Ovidi Nasonis Heroides, with the Greek Translation of Planudes. Edited by the late Arthur Palmer, Oxford, Clarendon 
Press. [Traduzione Italiana (con testo latino autonomo) di Giampiero Rosati: Lettere di eroine, Milano, Rizzoli/BUR, 1989]

\section{Letteratura critica}

BACHELARD, Gaston (1960): La poétique de la rêverie, Paris, PUF.

BAZzOCCHI, Marco Antonio (2009): «Aracoeli: il personaggio che si annulla», in: Marco Antonio Bazzocchi, Personaggi e romanzo nel Novecento italiano, Milano, Bruno Mondadori: 144 - 156.

D’ANGELI, Concetta (2003): Leggere Elsa Morante, Roma, Carocci.

DI FAZIO, Angela (2013): «Mito della crisi e crisi del mito in Aracoeli di Elsa Morante», in: Cuadernos de filología italiana 20/2013, pp. 17-35.

ELIAS, Norbert (1982 [1939]): Il processo di civilizzazione, Vol. 1, La civiltà delle buone maniere: la trasformazione dei costumi nel mondo aristocratico occidentale, Bologna, il Mulino.

GreIMAS, Algirdas Julien (1970): Du sens, Paris, Editions du Seuil.

JESI, Furio (1992): Letteratura e mito, Torino, Einaudi.

KERÉNYI, Károly (2001): Gli dei e gli eroi della Grecia, Il racconto del mito e la nascita della civiltà, Milano, il Saggiatore. [Traduzione italiana di Vanda Tedeschi dell'originale tedesco: Mythologie der griechen: die Götter- und Menschheitsgeschichten, Zürich, Rhein, 1951]

LEVI-STRAUSS, Claude (1968): Mythologiques III: L'origine des manières de table, Paris, Plon.

MARTÍNEZ GARRIDO, Elisa (eds.) (2003): Elsa Morante: la voce di una scrittrice e di una intellettuale aperta al secolo XXI, Madrid, Departamento de Filología Italiana UCM.

MARTÍNEZ GARRIDO, Elisa (2009): Between Italy and Spain. The tragedy of history and the salvific power of love in Elsa Morante and Maria Zambrano, in Sara Fortuna e Manuele Gragnolati ( a cura di), The Power of Disturbance. Elsa Morante's Aracoeli, Londra, Legenda, pp. 116-125.

PISCHEDDA, Bruno (2004): La grande sera del mondo. Romanzi apocalittici nell'Italia del benessere, Torino, Nino Aragno Editore.

RosA, Giovanna (1995): Cattedrali di carta. Elsa Morante romanziere, Milano, il Saggitore.

SERKOWSKA, Hanna (2002): «Percorsi androgini. Aracoeli: il romanzo definitivo di Elsa Morante», in: Il lettore di provincia 115.

SITI, Walter (1994): «Elsa Morante nell'opera di Pier Paolo Pasolini», in Studi Novecenteschi XXI/47-48 (numero speciale, intitolato: Vent'anni dopo 'La Storia'. Omaggio a Elsa Morante, a cura di Concetta D'Angeli e Giacomo Magrini): pp. 131-148.

UNTERSTEINER, Mario (1991 [1946]): La fisiologia del mito, Torino, BollatiBoringhieri. [edizione originale: Firenze, La Nuova Italia, 1946] 